\title{
Dynamic BER performance monitoring of WDM systems using a sum-of-Gaussian technique
}

\author{
B.Pal $^{1}$ and R.Gangopadhyay ${ }^{2}$ \\ ${ }^{1}$ Procyon Networks and Solutions, South Plainfield 07080 \\ email:biplab@procyonnet.com \\ ${ }^{2}$ Department of Electronics and Electrical Communication Engineering \\ Indian Institute of Technology, Kharagpur, India \\ Tel:+913222-78028,Fax:+913222-55303 \\ e-mail: ranjan@ece.iitkgp.ernet.in
}

Abstract: $\quad$ A sum-of-Gaussian (SGA) technique is presented for dynamic performance monitoring of WDM systems. The higher accuracy and usefulness of SGA technique over conventional Q-value analysis is established. It has been shown that Gaussian approximation $(\mathrm{GA})$ overestimates the transmission penalty by a large amount.

\section{INTRODUCTION}

With the advent of all optical networking, dynamic performance evaluation of optical digital transmission systems that are generally subjected to a large number of optical impairments is of extreme commercial interest because of automatic connection and fault management of the network [1]. In general, the power level, optical signal to noise ratio (OSNR) and the channel wavelength drift are measured at the input and output points of each network element like optical add/drop multiplexer, cross-connect etc. This kind of performance management, however, is inferior to the SONET layer physical performance monitoring of framer bytes $\mathrm{A} 1 / \mathrm{A} 2$ and parity bytes $\mathrm{B} 1 / \mathrm{B} 2$, because a large number of linear and non-linear effects in fiber that degrade the bit error rate (BER) of the system may not degrade the OSNR level at all. Hence Q value is normally proposed for dynamic channel monitoring [1]. However, forced usage of Q-value invites considerable inaccuracy in the prediction of system penalty. This is particularly true in situations handling inter-symbol interference (ISI), accumulated cross-talk, wavelength drift of optical channels, cascading effects of optical muxdemux etc. $[2,3]$.

In this article, we propose a simple and fast numerical technique based on sum-ofGaussian approximation (SGA) to represent accurately a non-Gaussian process of a time domain description of channel behavior available from optical channel monitor. We have 
developed an optimization algorithm to adapt this technique for BER evaluation. A Gaussian error index is also defined which quantifies the error in the estimation of BER when Q-value method is used.

\section{THEORETICAL ANALYSIS}

\subsection{Sum of Gaussian approximation}

Any non-Gaussian process defined by its probability density function (PDF) $W(x)$ can be expressed as a sum of $\mathrm{N}$ Gaussian PDFs each having a Gaussian PDF $W(x)=\sum_{i=1}^{N} c_{i} W_{G}^{i}\left(x, \sigma_{i}^{2}, \mu_{i}\right)$ where $c_{i}$ are the weights, $W_{G}^{i}\left(x, \sigma_{i}^{2}, \mu_{i}\right)$ is the 'i-th' Gaussian PDF with variance $\sigma_{i}{ }^{2}$ and mean $\mu_{i}$. To estimate the parameters $\sigma_{i}{ }^{2}$ and $\mu_{i}$, several stochastic methods and algorithms are available in practice based on minimization of the likelihood function $J=E_{x}\left(\frac{W(x)}{W_{A}(x)}\right)$, where $E_{x}($.$) denotes$ expectation operation and $W_{A}(x)$ is the PDF of the approximated process.

The estimation of the set $\left[c_{i}, \sigma_{i}{ }^{2}, \mu_{i}\right]$ requires solving $p$ sets of non-linear optimization equations by minimizing the norm of the error vector $E_{2 p}\left(\mu_{\mathrm{i}}, \sigma_{i}, c_{i}\right)=\frac{1}{p} \sqrt{\sum_{r=1}^{p} e_{2 r}{ }^{2}}$ with each error vector element obtained from the higher-order moments of the process: $e_{2 r}=\sqrt[2 p]{\frac{\left[M_{2 p}-M_{2 p}\left(c_{i}, \sigma_{i}\right)\right.}{(2 p-1) ! ! \sigma^{2}}}$ where $M_{2 p}$ is the actual $2 p$-th order moment of the process, $M_{2 p}\left(c_{i}, \sigma_{i}\right)$ is the approximated $2 \mathrm{p}$-th order moment of the process and $\sigma^{2}$ is the actual variance of the process. The error has been scaled by the Gaussian approximated $2 p$-th order moment of the process: $M_{2 p}(G A)=(2 p-1)(2 p-3) . .3 \cdot 1 \sigma^{2}$.

\subsection{The Algorithm}

In a practical system, it is normally sufficient to consider the number of terms in the sum-of-Gaussian approximation to be equal to four since in most cases a bit is affected 
by two adjacent bits contributing to the ISI process (which is likely to be distorted by 4 Gaussian processes). Hence, for a starting condition of the optimization, each of the $\sigma_{i}{ }^{2}$ is set to be the variance observed by the individual patterns (like 101,100 etc) and each pattern is considered to be equally likely. An iterative algorithm first optimizes the set $\left[c_{i}, \sigma_{i}{ }^{2}, \mu_{i}\right]$ based on the second-order moments and then gradually uses the higher-order moments of the process for final determination of the set $\left[c_{i}, \sigma_{i}{ }^{2}, \mu_{i}\right]$.

\subsection{Gaussian Error Index (GEI)}

We define a Gaussian error index (GEI) based on the calculation of $p$-th order moment for a non-Gaussian $W(x)$ as:

$$
G E I(p)=\frac{1}{2 p}\left[\sqrt{\sum_{r=1}^{p} \hat{e}_{2 r}^{2}(1)}+\sqrt{\sum_{r=1}^{p} \hat{e}_{2 r}^{2}(0)}\right]
$$

where we define the error vector element for 1 as

$$
e_{2 r}(1)=\sqrt[2 p]{\frac{\left[M_{2 p}(1)-(2 p-1) ! ! \sigma^{2}(1)\right.}{(2 p-1) ! ! \sigma^{2}(1)}}
$$

with $M_{2 p}(1)$ as the $2 p$-th order moment of the detected values of ' 1 ' and $\sigma^{2}(1)$ is the variance of the process ' 1 '. A high value of GEI is an indication that the process deviates from the Gaussian.

\subsection{Bit Error Rate Evaluation}

Once, $c_{i}, \sigma_{i}{ }^{2}$ are computed, the probability of error in detecting ' 1 ' is determined by $P_{e}(1)=0.5 \sum_{i=1}^{N} c_{i} \operatorname{erfc}\left(q_{i}\right)$ with $q_{i}=\frac{S_{t h}-\mu_{1}(1)}{\sigma_{1}(1)}$, where $S_{t h}$ is determined by the point of intersection of the PDF curves corresponding to ' 1 ' and ' 0 ' respectively obtained from the SGA method. The quantity $P_{e}(0)$ can be similarly calculated. 
Finally, the BER is calculated as $B E R=0.5 \times\left[P_{e}(1)+P_{e}(0)\right]$. The computation time with a $1 \mathrm{GHz}$ Pentium processor can be as low as below one second.

\section{RESULTS AND DISCUSSIONS}

Experiments have been conducted to validate the SGA method. Two kinds of situations have been considered: In the first case, the degraded eyes due to SPM, GVD and ASE noise have been generated due to transmission of $2^{8}-1$ bits NRZ signal over $50 \mathrm{Km}$ of SMF- 28 at $9,10,12$ and $14 \mathrm{dBm}$ launched power. In the second case, eyes are obtained when wavelength of the transmitter is drifted gradually from its ITU grid aligned position and the signal is obtained after it is passed through an equivalent 3-dB optical filter bandwidth of $20 \mathrm{GHz}$ centered at the transmitter ITU grid. In Figure 1, it can be seen that for both the cases, the sum-of-Gaussian method predicted BER is more close to the experimental BER and in the second case $Q$ value method is inapplicable because of high ISI content in the error statistics. In Figure 2, the mismatch between $\mathrm{Q}$, SGA predicted BER and actual BER is investigated with reference to GEI. It can be seen that at around $12 \mathrm{dBm}$ launched power, the error statistics has highest GEI (maximum non-Gaussian in nature) and then again it tends to be Gaussian. This is due to the significant influence of Raleigh scattering after $12 \mathrm{dBm}$ that drives the statistics to be Gaussian.
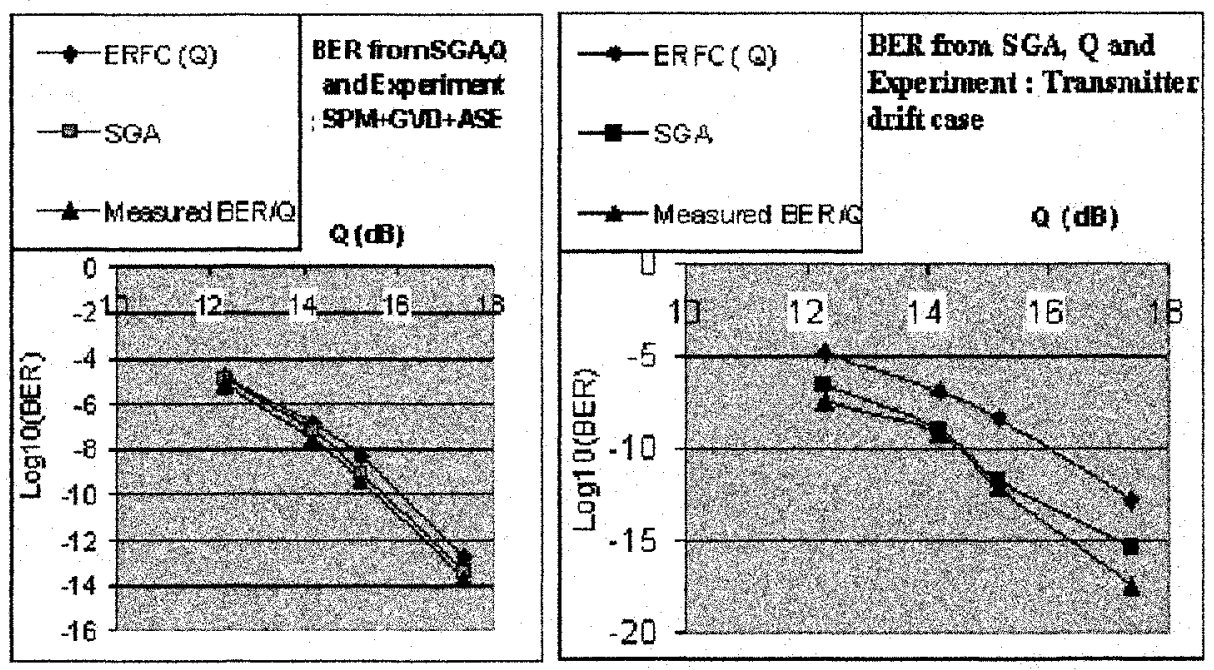

Figurel A comparison of BER obtained from $Q$ value and SGA method in GVD+SPM+ASE dominated and transmitter misalignment case 


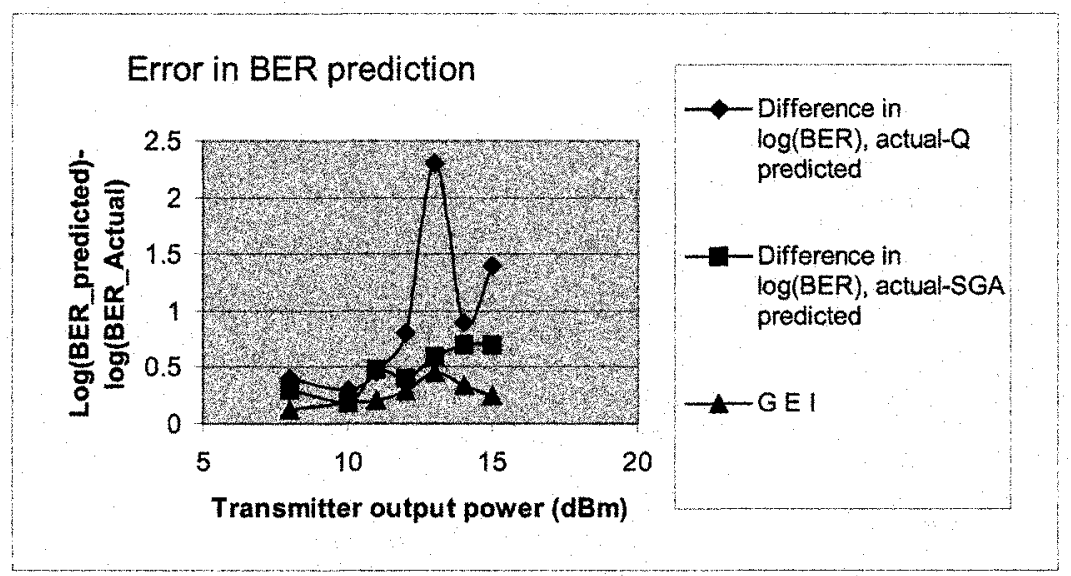

Figure 2 Error in predicting BER by SGA and Q value method for SPM+GVD+ASE noise case: Corresponding GEI values are also shown

\section{CONCLUSIONS}

The sum-of-Gaussian technique provides an efficient way to simulate the true PDF of the receiver's output statistics affected by additive Gaussian noise and ISI. It has been observed that the error in system penalty can be very large if we use Gaussian approximation for the receiver output with a GEI more than 0.3 . It is suggested that the Q-value technique may not be authentic for the receiver systems with a GEI more than 0.3 .

\section{REFERENCES}

[1] I. Shake and H. Takara, "Transparent and flexible performance monitoring using amplitude histogram method", OFC-2002, TuE P.19

[2] S. Betti, G. DeMarchis and E. Innone, Coherent Optical Communication System. John Wiley and Sons, 1995

[3] L. F. Ribereiro,J. R. F. Da Rocha, "Performance evaluation of EDFA preamplified receivers taking into account inter-symbol interference", IEEE J. of Lightwave Technol., vol.13, no.9, pp.225-232, 1995 